\title{
Charmed-Meson Decay Constants in Three-Flavor Lattice QCD
}

\author{
C. Aubin, ${ }^{1}$ C. Bernard,${ }^{2}$ C. DeTar, ${ }^{3}$ M. Di Pierro, ${ }^{4}$ E. D. Freeland,${ }^{5}$ Steven Gottlieb,${ }^{6}$ U. M. Heller,${ }^{7}$ \\ J. E. Hetrick ${ }^{8}$ A. X. El-Khadra, ${ }^{9}$ A. S. Kronfeld, ${ }^{10}$ L. Levkova, ${ }^{6}$ P. B. Mackenzie, ${ }^{10}$ D. Menscher, ${ }^{9}$ F. Maresca,${ }^{3}$ \\ M. Nobes,${ }^{11}$ M. Okamoto, ${ }^{10}$ D. Renner, ${ }^{12}$ J. Simone, ${ }^{10}$ R. Sugar,${ }^{13}$ D. Toussaint, ${ }^{12}$ and H. D. Trottier ${ }^{14}$ \\ (Fermilab Lattice, MILC, and HPQCD Collaborations) \\ ${ }^{1}$ Physics Department, Columbia University, New York, New York, USA \\ ${ }^{2}$ Department of Physics, Washington University, St. Louis, Missouri, USA \\ ${ }^{3}$ Physics Department, University of Utah, Salt Lake City, Utah, USA \\ ${ }^{4}$ School of Computer Science, Telecommunications and Information Systems, DePaul University, Chicago, Illinois, USA \\ ${ }^{5}$ Liberal Arts Department, The School of the Art Institute of Chicago, Chicago, Illinois, USA \\ ${ }^{6}$ Department of Physics, Indiana University, Bloomington, Indiana, USA \\ ${ }^{7}$ American Physical Society, Ridge, New York, USA \\ ${ }^{8}$ Physics Department, University of the Pacific, Stockton, California, USA \\ ${ }^{9}$ Physics Department, University of Illinois, Urbana, Illinois, USA \\ ${ }^{10}$ Fermi National Accelerator Laboratory, Batavia, Illinois, USA \\ ${ }^{11}$ Laboratory of Elementary-Particle Physics, Cornell University, Ithaca, New York, USA \\ ${ }^{12}$ Department of Physics, University of Arizona, Tucson, Arizona, USA \\ ${ }^{13}$ Department of Physics, University of California, Santa Barbara, California, USA \\ ${ }^{14}$ Physics Department, Simon Fraser University, Burnaby, British Columbia, Canada
}

(Dated: September 8, 2005)

\begin{abstract}
We present the first lattice QCD calculation with realistic sea quark content of the $D^{+}$-meson decay constant $f_{D^{+}}$. We use the MILC Collaboration's publicly available ensembles of lattice gauge fields, which have a quark sea with two flavors (up and down) much lighter than a third (strange). We obtain $f_{D^{+}}=201 \pm 3 \pm 17 \mathrm{MeV}$, where the errors are statistical and a combination of systematic errors. We also obtain $f_{D_{s}}=249 \pm 3 \pm 16 \mathrm{MeV}$ for the $D_{s}$ meson.
\end{abstract}

PACS numbers: $13.20 . \mathrm{Fc}, 12.38 . \mathrm{Gc}$

Flavor physics currently plays a central role in elementary particle physics [1]. To aid the experimental search for physics beyond the standard model, several hadronic matrix elements must be calculated nonperturbatively from quantum chromodynamics (QCD). One of the most important of these is the decay constant of the $B$ meson $f_{B}[2]$. Any framework for calculating $f_{B}$ should, therefore, be subjected to stringent tests, and such a test is a key aim of this Letter.

The most promising method for these nonperturbative calculations is numerical lattice QCD. For many years the results suffered from an unrealistic treatment of the effects of sea quarks. In the last few years, however, this obstacle seems to have been removed: with three flavors of sea quarks lattice QCD now agrees with experiment for a wide variety of hadronic quantities [3]. This validation of lattice QCD has been realized, so far, only for so-called "gold-plated" quantities: masses and matrix elements of the simplest hadronic states. Note, however, that many of the hadronic matrix elements relevant to flavor physics are in this class, including $f_{B}$.

The challenges in computing $f_{B}$ are essentially the same for the $D^{+}$-meson decay constant $f_{D^{+}}$. Experiments have observed the leptonic decay $D^{+} \rightarrow l^{+} \nu_{l}$, but not $B^{+} \rightarrow l^{+} \nu_{l}$. One can, thus, determine $\left|V_{c d}\right| f_{D^{+}}$, where $V_{c d}$ is an element of the Cabibbo-KobayashiMaskawa (CKM) matrix. Taking $\left|V_{c d}\right|$ from elsewhere, one gets $f_{D^{+}}$. In 2004 the CLEO-c Collaboration mea- sured $f_{D^{+}}$with a $20 \%$ error [4], and a more precise measurement is expected soon.

This Letter reports the first lattice-QCD calculation of $f_{D^{+}}$with three flavors of sea quarks [5]. We find

$$
f_{D^{+}}=201 \pm 3 \pm 6 \pm 9 \pm 13 \mathrm{MeV}
$$

where the uncertainties are statistical, and a sequence of systematic effects, discussed below. We also obtain the decay constant of the $D_{s}$ meson,

$$
f_{D_{s}}=249 \pm 3 \pm 7 \pm 11 \pm 10 \mathrm{MeV} .
$$

The second result is more precise than a recent latticeQCD calculation with the same sea quark content but non-relativistic heavy quarks, which found $f_{D_{s}}=290 \pm$ $20 \pm 41 \mathrm{MeV}$ [6]. These results are more reliable than older calculations [7] because we now incorporate (three) sea quarks and, for $f_{D^{+}}$, also because the light valence quark masses are smaller than before.

These results test the methods of Ref. [3] because they are predictions. The input parameters have been fixed previously [3, 8, 9, 10, 11], and, once comparably precise experimental measurements become available, one can see how Eqs. (11) and (2) fare. Indeed, this work is part of a program to calculate matrix elements for leptonic and semileptonic decays 10, 12, 13], neutral-meson mixing, and quarkonium [11, 14]. So far, these lattice QCD calculations agree with experiment for the normalization of 
TABLE I: Notation for quark masses used in this Letter.

\begin{tabular}{lll}
\hline \hline$m$ & \multicolumn{1}{c}{ Description } & \multicolumn{1}{c}{ Remark } \\
\hline$m_{c}$ & Charmed quark & From $m_{D_{s}}[10,11]$ \\
$m_{s}$ & Physical strange quark & From $m_{K}^{2}[8]$ \\
$m_{u}$ & Physical up quark & $m_{u}=m_{s} / 45.5[9]$ \\
$m_{d}$ & Physical down quark & $m_{d}=m_{s} / 19.6[9]$ \\
$m_{h}$ & Simulation's heavier sea quark & $m_{h} \approx 1.1 m_{s}$ \\
$m_{l}$ & Simulation's lighter sea quark & $0.1 m_{s} \leq m_{l} \lesssim 0.8 m_{s}$ \\
$m_{q}$ & Simulation's light valence quark & $0.1 m_{s} \leq m_{q} \lesssim m_{s}$ \\
\hline \hline
\end{tabular}

$D$-meson semileptonic form factors 12, 15, 16]. They also have predicted correctly the form-factor shape [12, 17], as well as the mass of the $B_{c}$ meson 14, 18].

In this set of calculations we use ensembles of unquenched lattice gauge fields generated by the MILC Collaboration [9, 19], with lattice spacing $a=0.175,0.121$, and $0.086 \mathrm{fm}$. The key feature of these ensembles is that they incorporate three flavors of sea quarks, one whose mass is close to that of the strange quark, and two with a common mass taken as light as possible.

For the sea quark and light valence quark we use the "Asqtad" staggered-fermion action [20]. Several different quark masses appear in this calculation; for convenience, they are defined in Table【 At $a=0.175,0.121$, and $0.086 \mathrm{fm}$ there are, respectively, 4,5 , and 2 ensembles with various sea quark masses $\left(m_{l}, m_{h}\right)$ 9, 19. The larger simulation mass, $m_{h}$ is close to the physical strange quark mass $m_{s}$. The light pair's mass $m_{l}$ is not as small as those of the up and down quark in Nature, but the range $0.1 m_{s} \leq m_{l} \lesssim 0.8 m_{s}$ suffices to control the extrapolation in quark mass with chiral perturbation theory $(\chi \mathrm{PT})$. For carrying out the chiral extrapolation, it is useful to allow the valence mass $m_{q}$ to vary separately from the sea mass 21. At $a=0.175,0.121$, and $0.086 \mathrm{fm}$ we have, respectively, 6,12 , and 8 or 5 values of the valence mass, in the range $0.1 m_{s} \leq m_{q} \lesssim m_{s}$.

A drawback of staggered fermions is that they come in four species, called tastes. The steps taken to eliminate three extra tastes per flavor are not (yet) proven, although there are several signs that they are valid. Calculations of $f_{D^{+}}$and $f_{D_{s}}$ are sensitive to these steps: if Eqs. (1) and (2) agree with precise measurements, it should be more plausible that the techniques used to reduce four tastes to one are correct.

For the charmed quark we use the Fermilab action for heavy quarks 22]. Discretization effects are entangled with the heavy-quark expansion, so we use heavy-quark effective theory (HQET) as a theory of cutoff effects 23]. This provides good control, as discussed in Ref. [24], and the framework has been tested with the (successful) prediction of the $B_{c}$ meson mass [14]. Nevertheless, heavyquark discretization effects are the largest source of systematic error in $f_{D_{s}}$, and the second-largest in $f_{D^{+}}$.

The decay constant $f_{D_{q}}$, for a $D_{q}$ meson with light valence quark $q$ and momentum $p_{\mu}$, is defined by [25]

$$
\left\langle 0\left|A_{\mu}\right| D_{q}\right\rangle=i f_{D_{q}} p_{\mu},
$$

where $A_{\mu}=\bar{q} \gamma_{\mu} \gamma_{5} c$ is an electroweak axial vector current. The combination $\phi_{q}=f_{D_{q}} \sqrt{m_{D_{q}}}$ emerges directly from the lattice Monte Carlo calculations. As usual in lattice gauge theory, we compute two-point correlation functions $C_{2}(t)=\left\langle O_{D_{q}}^{\dagger}(t) O_{D_{q}}(0)\right\rangle, C_{A}(t)=$ $\left\langle A_{4}(t) O_{D_{q}}(0)\right\rangle$, where $O_{D_{q}}$ is an operator with the quantum numbers of the charmed pseudoscalar meson, and $A_{4}$ is the (lattice) axial vector current. The operators are built from the heavy-quark and staggered-quark fields as in Ref. [26]. We extract the $D_{q}$ mass and the amplitudes $\left\langle D\left|O_{D_{q}}\right| 0\right\rangle$ and $\left\langle 0\left|A_{4}\right| D\right\rangle$ from fits to the known $t$ dependence. Statistical errors are determined with the bootstrap method, which allows us to keep track of correlations.

The lattice axial vector current must be multiplied by a renormalization factor $Z_{A_{4}^{c q}}$. We write [27] $Z_{A_{4}^{c q}}=$ $\rho_{A_{4}^{c q}}\left(Z_{V_{4}^{c c}} Z_{V_{4}^{q q}}\right)^{1 / 2}$, because the flavor-conserving renormalization factors $Z_{V_{4}^{c c}}$ and $Z_{V_{4}^{q q}}$ are easy to compute nonperturbatively. The remaining factor $\rho_{A_{4}^{c q}}$ should be close to unity because the radiative corrections mostly cancel [28]. A one-loop calculation gives [29] $\rho_{A_{4}^{c q}}=$ $1.052,1.044$, and 1.032 at $a=0.175,0.121$, and $0.086 \mathrm{fm}$. We estimate the uncertainty of higher-order corrections to be $2 \alpha_{s}\left(\rho_{A_{4}^{c q}}-1\right) \approx 1.3 \%$; $\alpha_{s}$ is the strong coupling.

The heart of our analysis is the chiral extrapolation, from the simulated to the physical quark masses. It is necessary, and non-trivial, because the cloud of "pions" surrounding the simulated $D_{q}$ mesons is not the same as for real pions. With staggered quarks the (squared) pseudoscalar meson masses are

$$
M_{a b, \xi}^{2}=\left(m_{a}+m_{b}\right) \mu+a^{2} \Delta_{\xi},
$$

where $m_{a}$ and $m_{b}$ are quark masses, $\mu$ is a parameter of $\chi \mathrm{PT}$, and the representation of the meson under the taste symmetry group is labeled by $\xi=P, A, T, V, I[30]$. A symmetry as $m_{a}, m_{b} \rightarrow 0$ ensures that $\Delta_{P}=0$. The "pion" cloud in the simulation includes all these pseudoscalars.

According to next-to-leading order $\chi \mathrm{PT}$ the decay constant takes the form

$$
\phi_{q}=\Phi\left[1+\Delta f_{q}\left(m_{q}, m_{l}, m_{h}\right)+p_{q}\left(m_{q}, m_{l}, m_{h}\right)\right],
$$

where $\Phi$ is a quark-mass-independent parameter. $\Delta f_{q}$ arises from loop processes involving light pseudoscalar mesons, and $p_{q}$ is an analytic function. To obtain them one must take into account the flavor-taste symmetry of the simulation [30] and the inequality (in general) of the valence and sea quark masses [21]. One finds [31]

$$
\Delta f_{q}=-\frac{1+3 g^{2}}{2\left(4 \pi f_{\pi}\right)^{2}}\left[\bar{h}_{q}+h_{q}^{I}+a^{2}\left(\delta_{A}^{\prime} h_{q}^{A}+\delta_{V}^{\prime} h_{q}^{V}\right)\right],
$$


where $f_{\pi} \approx 131 \mathrm{MeV}$ is the pion decay constant, $g$ is the $D-D^{*}-\pi$ coupling [32], and $\delta_{A}^{\prime}, \delta_{V}^{\prime}$ parametrize effects that arise only at non-zero lattice spacing [30]. The terms $\bar{h}_{q}$, $h_{q}^{I}, h_{q}^{A}$, and $h_{q}^{V}$ are functions of the pseudoscalar meson masses. The last two, $h_{q}^{A}$ and $h_{q}^{V}$, are too cumbersome to write out here. It is instructive to show the other two, $\bar{h}_{q}$ and $h_{q}^{I}$, when $m_{q}=m_{l}$ or $m_{h}$ :

$$
\begin{aligned}
\bar{h}_{q} & =\frac{1}{16} \sum_{\xi} n_{\xi}\left[2 I\left(M_{q l, \xi}^{2}\right)+I\left(M_{q h, \xi}^{2}\right)\right], \\
h_{l}^{I} & =-\frac{1}{2} I\left(M_{l l, I}^{2}\right)+\frac{1}{6} I\left(M_{\eta, I}^{2}\right), \\
h_{h}^{I} & =-I\left(M_{h h, I}^{2}\right)+\frac{2}{3} I\left(M_{\eta, I}^{2}\right),
\end{aligned}
$$

where $I\left(M^{2}\right)=M^{2} \ln M^{2} / \Lambda_{\chi}^{2}$ (with $\Lambda_{\chi}$ the chiral scale), and $M_{\eta, I}^{2}=\left(M_{l l, I}^{2}+2 M_{h h, I}^{2}\right) / 3$. The term $h_{q}^{I}$ receives contributions only from taste-singlet mesons (representation $I$ ). The term $\bar{h}_{q}$ receives contributions from all representations, with multiplicity $n_{\xi}=1,4,6,4,1$ for $\xi=P, A, T, V, I$, respectively. The analytic function is

$$
p_{q}=\left(2 m_{l}+m_{h}\right) f_{1}\left(\Lambda_{\chi}\right)+m_{q} f_{2}\left(\Lambda_{\chi}\right)+O\left(a^{2}\right),
$$

where $f_{1}$ and $f_{2}$ are quark-mass-independent parameters. They are essentially couplings of the chiral Lagrangian, and their $\Lambda_{\chi}$ dependence must cancel that of $\Delta f_{q}$. This specifies $O\left(a^{2}\right)$ terms proportional to $f_{1}$ and $f_{2}$, which can be removed after our fit. We estimate the remaining $O\left(a^{2}\right)$ effects of light quarks to be small: around $4 \%$ at $a=0.121 \mathrm{fm}$ and $1.4 \%$ at $a=0.086 \mathrm{fm}$.

The salient feature 33] of the chiral extrapolation of $\phi_{q}$ is that $\Delta f_{q}$ contains a "chiral $\log$ " $I\left(2 m_{q} \mu\right) \sim m_{q} \ln m_{q}$, which has a characteristic curvature as $m_{q} \rightarrow 0$. Equations (4) - (8) show that the chiral log is diluted by discretization effects, because $a^{2} \Delta_{\xi} \neq 0$ for $\xi \neq P$.

We can now discuss how we carry out the chiral extrapolation. Recall that we compute $\phi_{q}$ for many combinations of the valence and light sea quark masses. At each lattice spacing, we fit all results for $\phi_{q}$ to the mass dependence prescribed by Eqs. (40)-(10). Of the twelve parameters, eight $-\mu$, the four non-zero $\Delta_{\xi}, f_{\pi}, \delta_{A}^{\prime}$, and $\delta_{V}^{\prime}$-appear in the $\chi \mathrm{PT}$ for light pseudoscalar mesons. We constrain them with prior distributions whose central value and width are taken from the $\chi \mathrm{PT}$ analysis of pseudoscalar meson masses and decay constants on the same ensembles of lattice gauge fields $[9]$. The rest $-\Phi, g^{2}, f_{1}$, and $f_{2}$-appear only for charmed mesons. We constrain $g^{2}$ to its experimentally measured value, within its measured uncertainty [34]. Thus, only three parameters $-\Phi$, $f_{1}$, and $f_{2}$-are determined solely by the $\phi_{q}$ fit. To obtain physical results we reconstitute the fit setting the light sea quark mass $m_{l} \rightarrow\left(m_{u}+m_{d}\right) / 2$, and $\Delta_{\xi}=\delta_{A, V}^{\prime}=0$. For $\phi_{d}\left(\phi_{s}\right)$ we set the light valence mass $m_{q} \rightarrow m_{d}\left(m_{s}\right)$.

To isolate the uncertainties of the chiral extrapolation from other sources of uncertainty, we consider the ratio $R_{q / s}=\phi_{q} / \phi_{s}$. Figure 1 shows $R_{q / s}$ at $a=0.121 \mathrm{fm}$ as a function of $m_{q} / m_{s}$, projected onto $m_{q}=m_{l}$. The gray

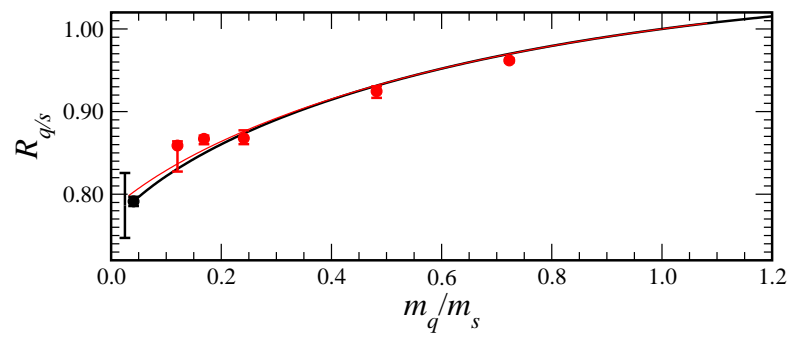

FIG. 1: Chiral extrapolation of $R_{q / s}$ at $a=0.121 \mathrm{fm}$. Data points show only statistical errors, but the systematic error of fitting is shown at left.

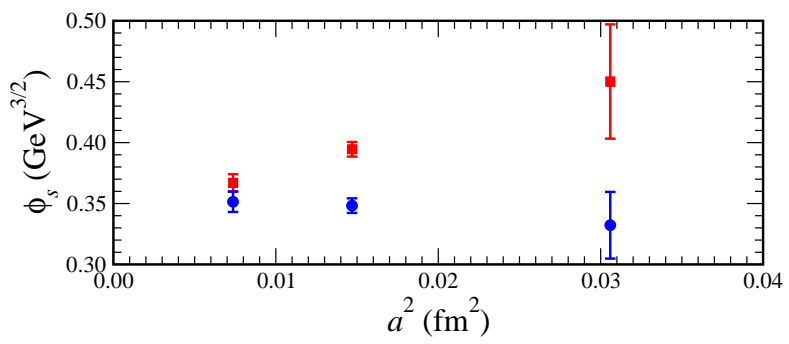

FIG. 2: Dependence of $\phi_{s}$ on $a^{2}$. Circles result from removing the $O\left(a^{2}\right)$ pieces in Eq. (10); squares omit this step.

(red) curve is the result of the full fit of $\phi_{q}$ to the separate sea- and valence-mass dependence. The black curve, and the extrapolated value at $m_{q} / m_{s}=0.05$, results from setting $\Delta_{\xi}=\delta_{A, V}^{\prime}=0$ when reconstituting the fit. At the other lattice spacings we obtain similar results.

The precision after the chiral extrapolation is, however, a bit illusory. We tried several variations in the fit procedure: fitting the ratio directly; adding terms quadratic in the quark masses to Eq. (10); variations in the widths of the prior constraints of the parameters. When these possibilities are taken into account, the extrapolated value of $R_{d / s}$ varies by $5 \%$, which we take as a systematic uncertainty. This variation could be reduced with higher statistics at the lightest sea quark masses.

The lattice spacing dependence of $\phi_{s}=f_{D_{s}} \sqrt{m_{D_{s}}}$ is shown in Fig. 2] The (blue) circles are the main results. In a preliminary report of this work [5] the $O\left(a^{2}\right)$ terms in $\phi_{s}$ were not removed. The (red) squares illustrate the effect of omitting this step. As one can see, the effect is small at $a=0.086 \mathrm{fm}$, but it is the main reason why the results in Eqs. (11) and (2) are smaller than in Ref. [5].

The $\chi \mathrm{PT}$ expressions for $\phi_{q}$ assume that the $D_{q}$ meson is static. Since its mass is around $1900 \mathrm{MeV}$ and the pseudoscalars are a few hundred $\mathrm{MeV}$, this is a good starting point. Some corrections to this approximation can be absorbed into the fit parameters, with no real change in the analysis. A more interesting change arises in the one-loop self-energy diagrams, for which the function $I\left(M^{2}\right)$ is modified, and depends on $m_{D^{*}}-m_{D}$ as well as $M$. By replacing our standard extrapolation by one using the modified function, we estimate the associated 
TABLE II: Error budget (in per cent) for $R_{d / s}, \phi_{s}, \phi_{d}$.

\begin{tabular}{lrrr}
\hline \hline source & $R_{d / s}$ & $\phi_{s}$ & $\phi_{d}$ \\
\hline statistics & 0.5 & 1.4 & 1.5 \\
input parameters $a$ and $m_{c}$ & 0.6 & 2.8 & 2.9 \\
higher-order $\rho_{A_{4}^{c q}}$ & 0 & 1.3 & 1.3 \\
heavy-quark discretization & 0.5 & 4.2 & 4.2 \\
light-quark discretization and $\chi \mathrm{PT}$ fits & 5.0 & 3.9 & 6.3 \\
static $\chi \mathrm{PT}$ & 1.4 & 0.5 & 1.5 \\
finite volume & 1.4 & 0.5 & 1.5 \\
\hline total systematic & 5.4 & 6.5 & 8.5 \\
\hline \hline
\end{tabular}

error to be $1.5 \%$ or less. Finite-volume effects also modify $I\left(M^{2}\right)$ : based on our experience with $f_{\pi}$ and $f_{K}[9]$ and on continuum $\chi \mathrm{PT}[35$, we estimate a further error of $1.5 \%$ or less.

Although $\chi \mathrm{PT}$ is able to remove (most of) the lightquark discretization errors, heavy-quark discretization effects remain. We estimate this uncertainty using HQET as a theory of cutoff effects [23, 24]. To arrive at a numerical estimate, one must choose a typical scale $\bar{\Lambda}$ for the soft interactions; we choose $\bar{\Lambda} \approx 500-700 \mathrm{MeV}$. We then estimate a discretization uncertainty of $2.7-4.2 \%$ at $a=0.086 \mathrm{fm}$. Similarly, the results at $a=0.121 \mathrm{fm}$ are expected to lie within $1-2 \%$ of those at $a=0.086 \mathrm{fm}$.

Because we cannot disentangle heavy- and light-quark discretization effects, to quote final results we average the results at $a=0.086$ and $0.121 \mathrm{fm}$. We then find

$$
\begin{aligned}
R_{d / s} & =0.786(04)(05)(04)(42) \\
\phi_{s} & =0.349(05)(10)(15)(14) \mathrm{GeV}^{3 / 2}
\end{aligned}
$$

which are the principal results of this work. The uncertainties (in parentheses) are, respectively, from statistics, input parameters $a$ and $m_{c}$, heavy-quark discretization effects, and chiral extrapolation. A full error budget is in Table【 all uncertainties are reducible in future work. The results for $f_{D^{+}}$and $f_{D_{s}}$ in Eqs. (1) and (2) are obtained via $f_{D_{s}}=\phi_{s} / \sqrt{m_{D_{s}}}, f_{D^{+}}=R_{d / s} \phi_{s} / \sqrt{m_{D^{+}}}$, by inserting the physical meson masses.

Present experimental measurements, $f_{D^{+}}=202 \pm 41 \pm$ $17 \mathrm{MeV}$ [4], $f_{D_{s}}=267 \pm 33 \mathrm{MeV}$ [25], are not yet precise enough to put our results in Eqs. (11) and (2) to a stringent test. The anticipated measurements of $f_{D^{+}}$and, later, $f_{D_{s}}$ from CLEO-c are therefore of great interest. If validated, our calculation of $f_{D^{+}}$has important implications for flavor physics. For $B$ physics it is crucial to compute the decay constant $f_{B}$. To do so, we must simply change the heavy quark mass. In fact, heavy-quark discretization effects, with the Fermilab method, are expected to be smaller, about half as big.

We thank the U.S. National Science Foundation, the Office of Science of the U.S. Department of Energy, Fermilab, and Indiana University for support, particularly for the computing needed for the project. Fermilab is operated by Universities Research Association Inc., under contract with the U.S. Department of Energy.

Note added: After this Letter was submitted, the CLEO-c Collaboration announced a new measurement, $f_{D^{+}}=223 \pm 16_{-9}^{+7} \mathrm{MeV}[36]$.

[1] See, for example, the CKM Unitarity Triangle Workshop, http://ckm2005.ucsd.edu/.

[2] V. Lubicz, Nucl. Phys. Proc. Suppl. 140, 48 (2005); M. Wingate, ibid. 140, 68 (2005).

[3] C. T. H. Davies et al., Phys. Rev. Lett. 92, 022001 (2004).

[4] G. Bonvicini et al., Phys. Rev. D 70, 112004 (2004).

[5] For a preliminary report of this work, see J. N. Simone et al., Nucl. Phys. Proc. Suppl. 140, 443 (2005).

[6] M. Wingate et al., Phys. Rev. Lett. 92, 162001 (2004).

[7] For example, A. X. El-Khadra et al., Phys. Rev. D 58, 014506 (1998); C. Bernard et al., ibid. 66, 094501 (2002).

[8] C. Aubin et al., Phys. Rev. D 70, 031504 (2004).

[9] C. Aubin et al., Phys. Rev. D 70, 114501 (2004).

[10] M. Di Pierro et al., Nucl. Phys. Proc. Suppl. 129, 328 (2004).

[11] M. Di Pierro et al., Nucl. Phys. Proc. Suppl. 129, 340 (2004).

[12] C. Aubin et al., Phys. Rev. Lett. 94, 011601 (2005).

[13] M. Okamoto et al., Nucl. Phys. Proc. Suppl. 140, 461 (2005); C. Aubin et al., in preparation.

[14] I. F. Allison et al., Phys. Rev. Lett. 94, 172001 (2005).

[15] M. Ablikim et al., Phys. Lett. B 597, 39 (2004).

[16] G. S. Huang et al., Phys. Rev. Lett. 94, 011802 (2005).

[17] J. M. Link et al., Phys. Lett. B 607, 233 (2005).

[18] D. Acosta et al., hep-ex/0505076

[19] C. Bernard et al., Phys. Rev. D 64, 054506 (2001); C. Aubin et al., ibid. 70, 094505 (2004).

[20] T. Blum et al., Phys. Rev. D 55, 1133 (1997); K. Orginos and D. Toussaint, ibid. 59, 014501 (1999); J. Lagäe and D. Sinclair, ibid. 59, 014511 (1999); G. P. Lepage, ibid. 59, 074502 (1999); K. Orginos, D. Toussaint and R. L. Sugar, ibid. 60, 054503 (1999); C. Bernard et al., ibid. 61, 111502 (2000).

[21] C. Bernard and M. Golterman, Phys. Rev. D 49, 486 (1994); S. R. Sharpe and N. Shoresh, ibid. 62, 094503 (2000).

[22] A. X. El-Khadra, A. S. Kronfeld and P. B. Mackenzie, Phys. Rev. D 55, 3933 (1997).

[23] A. S. Kronfeld, Phys. Rev. D 62, 014505 (2000).

[24] A. S. Kronfeld, Nucl. Phys. Proc. Suppl. 129, 46 (2004).

[25] S. Eidelman et al., Phys. Lett. B 592, 1 (2004).

[26] M. Wingate et al., Phys. Rev. D 67, 054505 (2003)

[27] A. X. El-Khadra et al., Phys. Rev. D 64, 014502 (2001).

[28] J. Harada et al., Phys. Rev. D 65, 094513 (2002).

[29] M. Nobes et al., private communication.

[30] W. Lee and S. Sharpe, Phys. Rev. D 60, 114503 (1999); C. Bernard, ibid. 65, 054031 (2002); C. Aubin and C. Bernard, ibid. 68, 034014 (2003); 68, 074011 (2003).

[31] C. Aubin and C. Bernard, Nucl. Phys. Proc. Suppl. 140, 491 (2005).

[32] B. Grinstein et al., Nucl. Phys. B 380, 369 (1992); J. L. Goity, Phys. Rev. D 46, 3929 (1992).

[33] A. Kronfeld and S. Ryan, Phys. Lett. B 543, 59 (2002). 
[34] A. Anastassov et al., Phys. Rev. D 65, 032003 (2002).

[35] D. Arndt and C.-J. Lin, Phys. Rev. D 70, 014503 (2004).

[36] M. Artuso et al., hep-ex/0508057 